\title{
Are the Results of the RE-LY Trial Reliable?
}

Dabigatran is an oral thrombin inhibitor that is indicated in Canada for the prevention of venous thromboembolism in patients who have undergone hip or knee replacement. ${ }^{1}$ In the RE-LY study (Randomized Evaluation of Long-term anticoagulant therapY), a 2-year multicentre non-inferiority trial, patients with atrial fibrillation who had an increased risk of stroke were randomly assigned (by allocation concealment) to receive dabigatran $110 \mathrm{mg}$ twice daily or $150 \mathrm{mg}$ twice daily (blinded) or warfarin (open-label). ${ }^{2}$ Concomitant use of acetylsalicylic acid (ASA, less than $100 \mathrm{mg} /$ day) and amiodarone was allowed. In addition, use of quinidine was permitted until 2 years after the trial started; at that point, the protocol was amended to limit use of this drug because of its ability to increase plasma concentrations of dabigatran. ${ }^{1,2}$

The authors of the RE-LY trial claimed that dabigatran was superior to warfarin at a dose of $150 \mathrm{mg}$ twice daily with respect to preventing stroke and systemic embolism. ${ }^{2}$ In addition, both the 110-mg and 150-mg doses were reported to be superior to warfarin with respect to the rate of hemorrhagic stroke. ${ }^{2}$ However, we have been unable to confirm the authors' conclusions because of flaws in the reported data and inadequacies in the reported components of the study.

The net clinical benefit (outcome) chosen for this trial, a composite of stroke, systemic embolism, pulmonary embolism, myocardial infarction, death, and major bleeding, encompasses problems typically seen with this class of medications, not those that are rare or yet to be discovered. Total serious adverse events were not reported, so the net effect of dabigatran cannot be assessed with certainty, especially given that there was a signal for increased risk of myocardial infarction relative to warfarin., ${ }^{2,3}$ Furthermore, although dabigatran $150 \mathrm{mg}$ twice daily appears to be non-inferior to warfarin with respect to major bleeding, this dose of the drug is associated with a statistically significant increase in the risk of life-threatening or non-life-threatening gastrointestinal bleeding relative to warfarin. ${ }^{2}$ The choice of the patient population is questionable, given the authors' report that nearly 6000 of the 18113 patients in the study had a CHADS(2) score of 0 or 1 . CHADS(2) is a risk stratification index describing the correlation between 5 known risk factors (congestive heart failure, 
hypertension, age greater than 75 years, diabetes, and previous stroke) and the risk of stroke in patients with atrial fibrillation., ${ }^{2,4}$ The evidence to support treating patients with these CHADS(2) scores is controversial; generally, patients are treated with warfarin only if the CHADS(2) score is above $2 .{ }^{5}$ Patients with CHADS(2) scores below 2 could be considered for ASA therapy; as such, the RE-LY trial should have excluded patients with CHADS(2) score below 2 or should have considered an ASA arm.

The authors' definition of systemic embolism did not detail if screening was mandatory for all patients or if the reported systemic emboli were from symptomatic or asymptomatic patients. Screening for asymptomatic events would increase the number of systemic emboli, regardless of their clinical relevance. It is also unclear from the data in Table 2 of the report ${ }^{2}$ the number of patients who had at least 1 systemic embolus, a fatal stroke, or fatal bleeding. Creatinine clearance was neither measured nor monitored during the study. ${ }^{2}$ Dabigatran is subject to primarily renal clearance $(85 \%)$, and the degree of renal impairment is proportional to the extent of exposure to the drug. ${ }^{1}$ In patients with creatinine clearance below $30 \mathrm{~mL} / \mathrm{min}$, dabigatran is contraindicated, as plasma concentrations of the drug will be 6 times higher than in patients with normal renal function; in patients with creatinine clearance of $30-50 \mathrm{~mL} / \mathrm{min}$, concentrations will be 2.7 times higher. ${ }^{1}$ Therefore, patients in the dabigatran arms with moderate renal impairment could have experienced an increase in therapeutic effect or an increase in the risk of serious adverse events relative to patients in the warfarin arm. ${ }^{1}$

Use of amiodarone, a drug that can increase plasma concentrations of dabigatran by $50 \%$, was evenly distributed among the treatment groups; however, use of quinidine, a drug that is contraindicated for use with dabigatran because it can increase dabigatran concentrations by over $100 \%$, was not noted in the baseline characteristics, and the distribution was not noted for each treatment arm., ${ }^{2,5}$ Once again, increased concentrations of dabigatran could have produced an increased therapeutic effect in terms of reducing coagulation but also increasing the risk for adverse effects. ${ }^{1,2,6}$ The selection of patients for the RE-LY study was done according to intention-to-treat principles; however, non-inferiority trials require a per-protocol analysis to confirm the non-inferiority that is observed with an intention-to-treat analysis. ${ }^{7}$ This had little impact in relation to the comparison between warfarin and dabigatran $150 \mathrm{mg}$ and the finding of superiority; technically, a per-protocol analysis should have been part of the prespecified statistical plan.

We have contacted the corresponding author of the RE-LY study seeking clarification of these issues but have received no response to date. How RELY-able, then, are the claims made by the authors?

\section{References}

1. Pradax [monograph]. In: Repchinksy C, editor. Compendium of pharmaceuticals and specialties [online]. Ottawa (ON): Canadian Pharmacists Association; 2009 [cited 2009 Oct 20]. Available from: http://e-therapeutics.ca. Subscription required to access content.

2. Connolly SJ, Ezekowitz MD, Yusuf S, Eikelboom J, Oldgren J, Parekh A, et al.; RE-LY Steering Committee and Investigators. Dabigatran versus warfarin in patients with atrial fibrillation. $N$ Engl J Med 2009; 361(12):1139-1151.

3. Serious adverse event analysis: lipid-lowering therapy revisited. Ther Lett [serial on Internet] 2001 Aug-Oct [cited 2009 Sep 17];42:1-2. Available from: www.ti.ubc.ca/PDF/42.PDF

4. Gage BF, Waterman AD, Shannon W, Boechler M, Rich MW, Radford MJ. Validation of clinical classification schemes for predicting stroke: results from the National Registry of Atrial Fibrillation. JAMA 2001;285(22):2864-2870.

5. Cardiovascular disorders: supraventricular tachycardia. In: Gray J, editor. Therapeutic choices. 5th ed. Ottawa (ON): Canadian Pharmacists Association; 2003. p. 531-543.

6. Pradax (dabigatran etexilate) [prescribing information]. Burlington (ON): Boehringer Ingelheim Canada Ltd; 2008 Jun.

7. Scott IA. Non-inferiority trials: determining whether alternative treatments are good enough. Med J Aust 2009;190(6):326-330.

\section{Matthew P Tsang, BSc(Pharm)}

Aaron Tejani, BSC(Pharm), PharmD, ACPR

I fan Kuo, BSC(Pharm), ACPR, PharmD

Burnaby Hospital

Fraser Health Authority

Burnaby, British Columbia

Matthew Tsang was a Pharmacy Resident with the Fraser Health Authority in 2009/2010, when this letter was prepared.

Advertisers' Index

\begin{tabular}{lll}
\hline & Ad Page & Prescribing Information \\
\hline Amgen / Neulasta & 100 & $163-165$ \\
\hline Health Pro / Corporate & 137 & - \\
\hline Hospira / Precedex & 96 & 157,158 \\
\hline Pfizer / Fragmin & IBC & $171-175$ \\
\hline Pfizer / Sutent & 103 & $166-170$ \\
\hline Pharmaceutical Partners of Canada / Safety Ad & IFC & - \\
\hline Pharmaceutical Partners of Canada / Safety Ad & OBC & - \\
\hline Sandoz / Corporate & 94 & - \\
\hline Sanofi-Aventis / Plavix & 99 & $159-162$
\end{tabular}

